\section{Dynamic multicasting in wavelength-division multiplexing ring employing multicast- capable optical add/drop multiplexers}

\author{
Yunfeng Peng, Weisheng Hu, Xiaodong Wang, \\ Weiqiang Sun, Yaohui Jin, and Wei Guo \\ Shanghai Jiao Tong University, The State Key Laboratory \\ on Fiber-Optic Local Area Networks and Advanced \\ Optical Communication Systems, Electronic Department, \\ 800 Dongchuan Road, Minhang, Shanghai, 200240, \\ China \\ E-mail: wshu@sjtu.edu.cn
}

\begin{abstract}
We analyze the multicast capability of a multicast-capable optical add/drop multiplexer (MC-OADM) and investigate the dynamic network performance of the MC-OADM ring in comparison with that of the normal OADM ring without multicast capability. The simulation results show that the multicast scheme using MC-OADMs provides more multicast services and occupies fewer resources. (c) 2006 Society of Photo-Optical Instrumentation Engineers.

[DOI: 10.1117/1.2280496]
\end{abstract}

Subject terms: optical fiber communications; optical networks; optical multicasting; multicast-capable optical add/drop multiplexer.

Paper 060193LR received Apr. 3, 2006; revised manuscript received Jun. 9, 2006; accepted for publication Jun. 20, 2006; published online Aug. 16, 2006.

\section{Introduction}

It becomes clear that the broadband video such as interactive digital television (DTV) and high-definition television (HDTV) are among the most important applications in the next-generation networks. These applications require broad bandwidth together with multicast function to deliver the shared streams to multiple receivers more efficiently. The existing pure Internet protocol (IP) store-and-forward delivery may be inefficient due to the considerable processing delay through multihop routers. ${ }^{1}$ Alternatively, multicasting can be implemented on wavelength-division multiplexing (WDM) networks on the basis of transparent connectionoriented delivery. ${ }^{1,2}$

Recently, multicasting was extensively investigated in WDM mesh networks. ${ }^{2}$ In mesh topology, each node is connected by multicast-capable optical cross-connects (MC-OXCs) in which large dimension splitters are used to construct light trees. ${ }^{3}$ Actually, ring topology is widely used in the carrier's optical network. The normal optical add/ drop multiplexers (OADMs) employed in current ring perform only add/drop or bypass of the signals without multicast function. Multicast-capable access node architectures using splitters and $2 \times 2$ switches were proposed to support multicasting in the slotted photonic ring network. ${ }^{4}$ These architectures can be improved to be multicast-capable

0091-3286/2006/\$22.00 @ 2006 SPIE
OADMs (MC-OADMs). A broadcast and select OADM was used as normal OADM in Ref. 5, and could be improved to be an MC-OADM. Using MC-OADMs, more destination nodes on a ring can drop a portion of optical power without destroying the transparency of the trunk traffic.

In this letter, we investigate the multicast capability of an MC-OADM and the network performance of the MCOADM ring with dynamic wavelength connections of both multicast and unicast. It is assumed that all lower rate traffic have been groomed and aggregated into wavelength channels at source nodes using electronic multiplexing techniques. The simulation results show that the MCOADM scheme provides more connections (lower blocking probability) and occupies less bandwidth resources in comparison with the scheme using normal OADM.

\section{Architecture of MC-OADM}

Figure 1 illustrates the schematic of the MC-OADM architecture. It consists of preamplifiers, multiplexers (MUXs), multicast add/drop elements (MADEs), demultiplexers (DMUXs), and booster amplifiers along the signal transmission direction. The preamplifiers and booster amplifiers compensate the power loss caused by fiber links and passive components inside the MC-OADM node. Each channel disassembled by the DMUXs from the input fiber is equipped with a MADE. Each MADE consists of a $1 \times 2$ splitter with coupling ratio of 90:10 or other, an on/off gate, and a $2 \times 1$ switch. Only a small fraction (e.g., 10\%) of the input optical power is tapped and dropped to the local access station while the remaining large fraction (e.g., 90\%) of the input power is passed by the MC-OADM. The on/off gates are used to receive or stop the tapped signals. The 2 $\times 1$ switches are used to select either the passing signals or the adding signals. Together with the cooperation of on/off gate, $2 \times 1$ switch, transmitter $(\mathrm{Tx})$ and receiver $(\mathrm{Rx})$, the

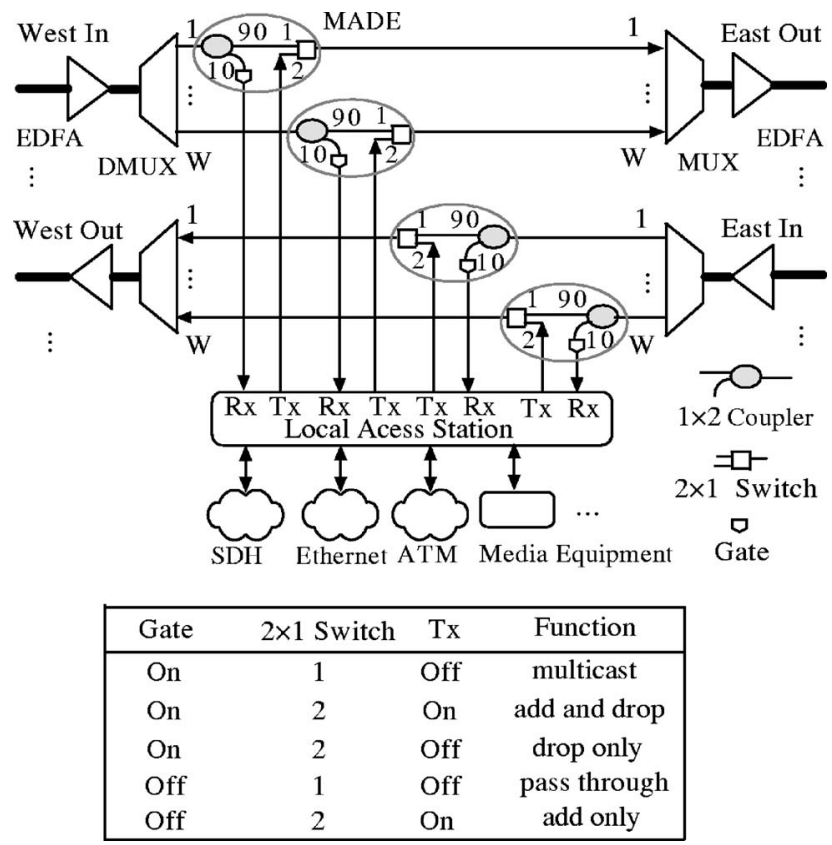

Fig. 1 Illustration of MC-OADM architecture and its operation states (EDFA: erbium-doped fiber amplifier). 
MADE works in five states: multicast, add and drop, drop only, pass by, and add only, as listed in Fig. 1. Using such MC-OADM, a bus-like optical traffic "pipe" (light tree) can be set up for a multicast group, by which any destination can receive the traffic carried on the pipe without destroying the transparency of the trunk traffic.

It should be noted that the electronic devices located at the local access station will aggregate the branch traffic from local Ethernet, asynchronous transfer mode (ATM), synchronous digital hierarchy (SDH), media equipment, etc. into a wavelength capacity, and thus we address only the traffic at a wavelength granularity level.

\section{Dynamic Network Performance Evaluation}

\subsection{Problem Description}

Like multicasting in the WDM mesh networks, a multicast routing and wavelength assignment (MC-RWA) problem also exists for multicasting in WDM ring. ${ }^{2,6}$ Let $R(V)$ be the bidirectional WDM ring, where $V$ denotes the set of network nodes. Let $Q(s, D)$ be an optical multicast request, where $D$ denotes the set of destination nodes, and $s$ ( $s$ $\in V, s \notin D)$ denotes the source node. The functionality of MC-RWA is to find one or more wavelength channels along either clockwise or counterclockwise of $R(V)$ to connect $s$ to nodes in $D$. The wavelength channels can be light paths (point to point wavelength channels) or light trees (point to multipoints wavelength channels). The existing shortest path tree (SPT) and minimum spanning tree (MST) heuristics can also be introduced into WDM ring. ${ }^{6}$ The SPT minimizes the delay from $s$ to every destination node in $D$, while the MST minimizes the network cost in terms of the total links costs of a routing tree. Figures 2(a) and 2(b) illustrate an example of the SPT tree and the MST tree for a multicast group with $s=1$ and $D=\{3,4,6\}$.

Since normal OADMs have no multicast capability to set up light trees for $Q(s, D)$, we use $|D|$ different light paths originating from $s$ to simulate a light tree; that is, for each node pair of $(s, d)$ where $d \in D$, we try to find the shortest path along clockwise or counterclockwise direction under constraints of wavelengths and add/drop ports availability. An example is shown in Fig. 2(c) for the multicast group $Q(1,\{3,4,6\})$. We call this scheme the normal scheme (N-Scheme).

As for multicasting in the ring employing MC-OADMs, we first divide $|V|-1$ nodes in $V$ except $s$ into two half rings according to $s$. Considering the ring in Fig. 2 where the source node $s=1$, nodes $2,3,4$, and 5 fall into the right half ring while nodes 8,7 , and 6 fall into the left half ring. The

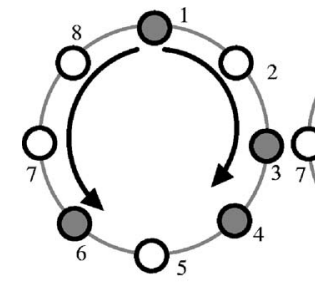

(a)

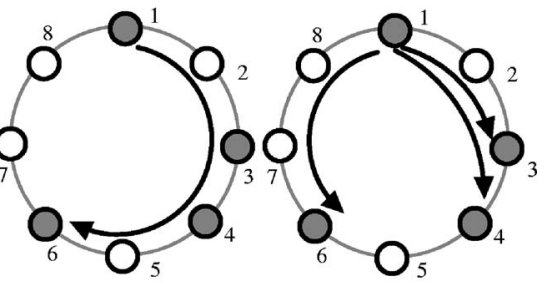

(b)

(c)
Fig. 2 Example of light trees: (a) SPT, (b) MST, and (c) multicasting in WDM ring employing normal OADM. destination nodes falling into the right half ring are routed in clockwise within the right half ring and those falling into the left half ring are routed in counterclockwise confined in the left half ring. Using this method, SPT trees can be built. We call this scheme multicast scheme (M-Scheme).

As in the mesh topology, there are also two definitions to evaluate service blocking for dynamic multicasting in WDM ring, i.e., session (a multicast group) blocking and member (destination) blocking. For a multicast request $Q(s, D)$, if there is any member in $D$ being rejected due to no available wavelength channel or no available drop port, all members in $D$ are rejected, which is called session blocking. When only the rejected members are counted, we call it member blocking which is adopted in this work.

\subsection{Performance Simulation and Analysis}

We consider both unicast and multicast connections and suppose $P \%(0<P<100)$ of the requests are multicast ones. Note $|D|$ uniformly changes from 2 up to $|V|-1$ among the multicast requests. All requests arrive according to a Poisson process with rate $\lambda$ and the holding time of each request is exponentially distributed with a mean $1 / \mu$, i.e., the load measured in Erlangs is $\lambda / \mu$. Totally $10^{6}$ requests (both unicast and multicast ones) are considered at each load and the member blocking probability is calculated on these $10^{6}$ requests. The resource consumption is calculated in terms of wavelength segments consumed per session. (A wavelength segment is a wavelength channel between two successive nodes along the ring.) The calculated member blocking probability and the wavelength segments consumption at each load on a 10 node ring with three traffic scenarios: 20,50 , and $70 \%$ of $10^{6}$ requests being multicast requests, are illustrated in Fig. 3. (There are two fibers at each direction of the ring and 16 wavelengths in each fiber.)

Figure 3(a) shows the blocking probability of the $\mathrm{N}$-Scheme at light load is lower than that of the M-Scheme. However, as the load increases, the blocking probability of the N-Scheme increases dramatically and goes higher than that of the M-Scheme. The reasons are that, first, in the RWA for the N-Scheme, if a light path cannot be found in the clockwise (counterclockwise) direction, the RWA will try to find another lightpath in the anticlockwise (clockwise) direction, nevertheless in the RWA for the M-Scheme to construct SPT tree, the light path searching is confined to the clockwise half ring or the counterclockwise half ring rather than the entire ring; second, the RWA for the $\mathrm{N}-\mathrm{Scheme}$ can find a light path longer than the half ring while the RWA for the M-Scheme always finds a light path no longer than the half ring; third, more than one destination can share the same lightpath to form SPT tree in M-Scheme by using MADEs, i.e., the M-Scheme consumes less resources than the $\mathrm{N}-\mathrm{Sch}$ eme does, as can be seen from Fig. 3(b), where the wavelength segments consumed per session for the $\mathrm{N}$-Scheme is larger than that for the M-Scheme, on all three traffic scenarios. Due to these three features, the available wavelength and add/drop ports are sufficient at light load so that the N-Scheme has more chances to find a light path than the M-Scheme does. But at heavy load, since the available resources get severe for the $\mathrm{N}$-Scheme, the blocking probability of the N-Scheme rises sharply and becomes unacceptable 0.1 at 13 erlangs in the 


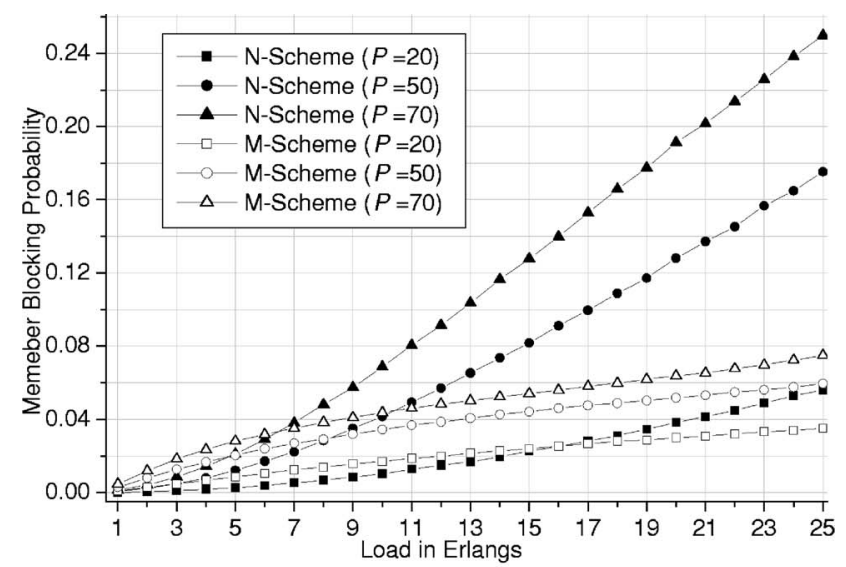

(a)

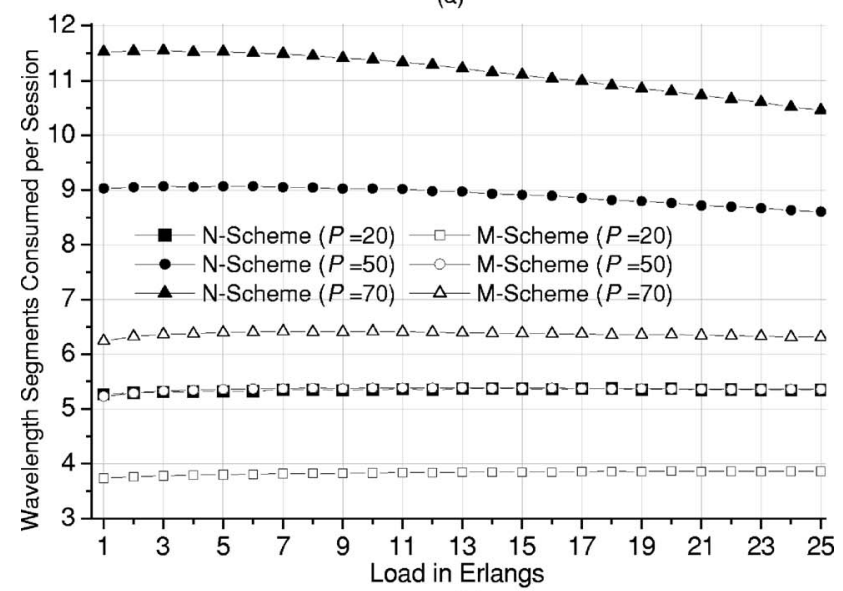

(b)

Fig. 3 Network performance of MC-OADM ring: (a) blocking probability, and (b) wavelength consumption.

scenario of $70 \%$ of $10^{6}$ requests being multicast requests and $30 \%$ being unicast ones, while that of the M-Scheme remains under 0.08 at 25 erlangs.

Figure 3 also shows that the member blocking probability and the wavelength segments consumed per session will increase as the percentage of multicast requests increasing for both the $\mathrm{N}$-Scheme and the M-Scheme. The reason is that the more multicast requests arrive, the more destination nodes must be connected, then the more resources are occupied, and eventually, the more members are blocked due to the severe resources availability.

\section{Conclusion}

It is expected that the next-generation optical network will be updated to support multicasting services, including the widely used ring topology. MC-OADMs will be the key node equipment in the optical multicasting ring. A typical MC-OADM architecture was analyzed in this letter. The dynamic network performance of the MC-OADM ring was compared with that of the normal OADM ring without multicasting capability. Simulation results show the MCOADM scheme outperforms the normal scheme in terms of destination blocking probability and wavelength consumption. The blocking performance of the MC-OADM ring at light load can be improved by modifying its RWA. For example, if there are some destination nodes failing to be reached using the SPT trees, MST trees can be used.

\section{Acknowledgment}

This work is supported by the National Natural Science Foundation of China (NSFC), 863 Program, the Ministry of Education of China, and the Shanghai Sci \& Tech Committee.

\section{References}

1. C. Qiao, M. Jeong, A. Guha, X. Zhang, and J. Wei, "WDM multicasting in IP over WDM networks," in Proc. IEEE Int'l Conf. on Network Protocols (ICNP99), Toronto, Canada, pp. 89-96 (1999).

2. J. He, S.-H. G. Chan, and D. H. K. Tsang, "Multicasting in WDM networks," IEEE Commun. Surv. Tut. 4(1), 2-20 (2002).

3. W. S. Hu, and Q. J. Zeng, "Multicasting optical cross connects employing splitter-and-delivery switch," IEEE Photon. Technol. Lett. 10(7), 970-972 (1999).

4. S. Aleksic and K. Bengi, "Multicast-capable access nodes for slotted photonic ring networks," in Proc. Eur. Conf. Opti. Commun. (ECOC 2000), Munich, Germany, Vol. 3, pp. 83-84 (2000).

5. A. Boskovic, M. Sharma, N. Antoniades, and M. Lee, "Broadcast and select OADM nodes: application and performance trade-offs," in Proc. Opti. Fiber Commun. Conf. (OFC 2002), Anaheim, CA, pp. 158-159, (2002).

6. X. Jia, X. Hu, L. Ruan, and J. Sun, "Multicast routing, load balancing, and wavelength assignment on tree of rings," IEEE Commun. Lett. 6(2), 79-81 (2002). 